\title{
Novel synthesis of superparamagnetic plasmonic core-shell iron oxide-gold nanoparticles
}

\author{
Arne Billen ${ }^{1}$, Amaury de Cattelle ${ }^{2}$, Johanna K. Jochum ${ }^{3}$, Margriet J. Van Bael ${ }^{3}$, Johan Billen ${ }^{4}$, \\ Jin Won Seo ${ }^{5}$, Ward Brullot ${ }^{1}$, Guy Koeckelberghs ${ }^{2}$, and Thierry Verbiest ${ }^{1}$
1. Department of Chemistry, Laboratory for Molecular Electronics and Photonics, KU Leuven, Celestijnenlaan 200D, Box 2425, 3001 Leuven, Belgium; arne.billen@kuleuven.be (A.B.); ward.brullot@kuleuven.be (W.B.); thierry.verbiest@fys.kuleuven.be (T.V.)
2. Department of Chemistry, Laboratory for Polymer Chemistry and Materials, KU Leuven, Celestijnenlaan 200F, Box 2404, 3001 Leuven, Belgium; amaury.decattelle@kuleuven.be (A.D.C); guy.koeckelberghs@kuleuven.be (G.K.)
3. Department of Physics and Astronomy, Laboratory of Solid State Physics and Magnetism, KU Leuven, Celestijnenlaan 200D, 3001 Leuven, Belgium; johanna.jochum@kuleuven.be (J.K.J.); margriet.vanbael@kuleuven.be (M.J.V.B.)

\section{Department of Biology, Ecology, Evolution and Biodiversity Conservation Section, KU Leuven, Naamsestraat 59, Box 2466, 3000 Leuven, Belgium; johan.billen@kuleuven.be (J.B.)}

5. Department of Materials Engineering, KU Leuven, Kasteelpark Arenberg 44, 3001 Leuven, Belgium; maria.seo@kuleuven.be (J.W.S)

\begin{abstract}
Magnetically removable plasmonic nanoparticles are of great interest for biomedical and catalytical applications. A novel and straightforward method to prepare a coreshell structure consisting of a superparamagnetic $\mathrm{Fe}_{3} \mathrm{O}_{4}$ core coated with a plasmonic gold shell is described. The synthesis combines ease of functionalization, limited supervision and high reproducibility while being environmentally friendly. A gold shell with a tunable thickness of 30 to $70 \mathrm{~nm}$ was grown on a $\mathrm{Fe}_{3} \mathrm{O}_{4}$ core $20 \mathrm{~nm}$ in diameter through reduction of gold salt in an ultrasonic bath. The thickness of the gold shell could be tuned by varying the amount of gold salt added. It was possible to prepare core-shell nanoparticles of sizes between 80 and $160 \mathrm{~nm}$. The magnetic and plasmonic behavior of the core-shell nanoparticles is studied. To demonstrate potential use in biomedical applications, a functionalization with polyethylene glycol (PEG) is performed.
\end{abstract}

\section{Keywords}

Core-shells; magnetic; plasmonic; environmentally friendly; tunable

\section{Introduction}

Iron oxide nanoparticles (IONP) have been used in several biomedical applications. These include drug delivery, magnetic resonance imaging (MRI) contrast agent and hyperthermia treatments, among other applications [1,2][3]. Combining this magnetic property with a plasmonically active metal shell could open up even more applications, such as laser-induced hyperthermia, biosensing and radiotherapy, among others [4]. 
Gold seems the most promising choice for the metal shell. To date, gold has been the preferred coating material amongst others because the optical qualities are well-known [5]. Gold nanoparticles absorb in the visible-near infrared region and they are also easily functionalized, especially when using amines and thiols [6][4,7]. The inert nature of gold protects the nanoparticles from oxidation, while also making them suitable for biological environments and inducing no or lowered toxicity, though this is a topic under debate[8]. Literature suggests that toxicity is also a function of the functionalization of the surface, for example, PEG-functionalization appears to lower toxicity[9]. In general, iron oxide-gold core-shell nanoparticles are of great interest and can be useful for both imaging (diagnostics) and therapeutic applications. This particular combination has been coined "theranostics", indicating the multifunctional character of the composite nanoparticle[10].

Many pathways have been found for the synthesis of iron oxide-gold core-shell nanoparticles[11-15]. At its core, these syntheses all have one thing in common: the reduction of a gold salt in solution to form a gold shell around the core. Many syntheses utilize very small gold nanoparticles, referred to as gold seeds, as a starting point for the growth of the gold shell[16,17]. The use of gold seeds infers some drawbacks; since these gold seeds need to bind to the iron oxide, usually using amine groups on the iron oxide, crosslinking between several core particles will occur. This induces clustering of the nanoparticles, which will at best decrease solubility and at worst renders the nanoparticles insoluble. The gold salt and reducing agent also have to be added in small iterations to achieve the best results, which lowers batch size and is more labor intensive.

Direct deposition of gold onto iron oxide nanoparticles has been reported in organic solvents[18,19][11]. The first two methods mentioned used small magnetic cores $(5 \mathrm{~nm})$ which are hard to separate magnetically because of the relatively low magnetic force. Methods that involve organic solvents are inherently less environmentally friendly.

Still, the search is on for a synthesis that combines large yields, limited supervision, reproducibility and smooth gold shells, all while being environmentally friendly. The core-shells also require ease of functionalization, which is largely dependent on the ligands that are present on the gold surface after synthesis. Presented here is a novel synthesis method that produces well dispersed gold shells around iron oxide nanoparticles in an aqueous solution without iterative addition of reagents. A schematic view of the steps required for the synthesis is shown below in figure 1 . The use of an ultrasonic bath removes the need for stirring, while also reducing nanoparticle aggregation in crucial parts of the gold shell formation. Yields up to $10 \mathrm{mg}$ per batch can be achieved. The only non-environmentally friendly reagent used in this synthesis is formaldehyde, though it can be omitted from the reaction. Omitting formaldehyde yields slightly worse solubility of the core-shell nanoparticles and is discussed in more detail in supporting material S1.

The core-shell nanoparticles as prepared are water soluble. The thickness of the gold shell can be tailored by varying the amount of gold salt added. Using thiolated PEG as a ligand, the solubility in water and the biocompatibility can be further enhanced. 


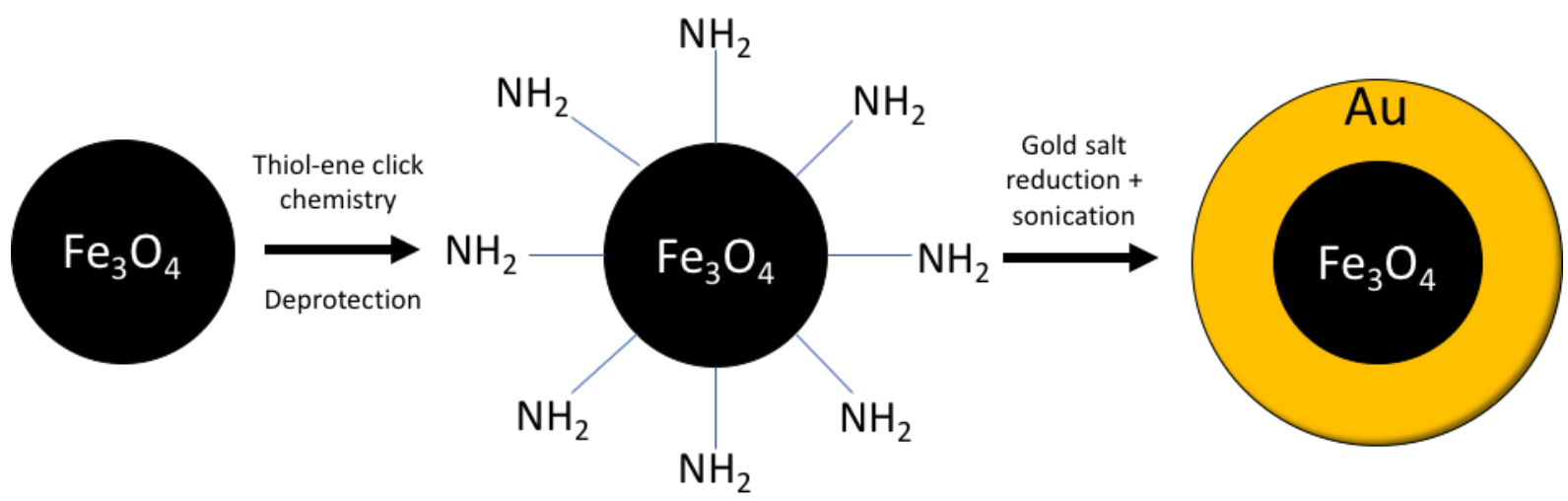

Figure 1: schematic view of the preparation steps required and the direct gold shell deposition

\subsection{Materials and methods}

\subsection{Synthesis}

\subsubsection{Superparamagnetic iron oxide nanoparticles (IONPs)}

Iron oxide nanoparticles (IONPs) with a diameter of around $20 \mathrm{~nm}$ are synthesized following the protocol of Chen et al[20]. The IONPs are amine-functionalized following the protocol of Bloemen et al[21]. A TEM picture of the as synthesized IONPs is provided in supporting material S2.

The synthesis of IONPs is done by two separate reactions. First an iron-oleate precursor is created. This precursor is then thermally decomposed into the IONP[22]. We can tune the size of the IONPs by varying the heating rate of the thermal decomposition.

The iron-oleate precursor is prepared by adding sodium-oleate (27.375 g, Sigma Aldrich) and iron(III)chloride (8.1 g, Labo Scientific) to a solution of MilliQ water (45 $\mathrm{ml})$, ethanol (60 ml, absolute, Fisher Scientific) and heptane (105 ml, 99\%, Fisher Scientific). The resulting mixture is refluxed for 4 hours at a temperature of $74^{\circ} \mathrm{C}$. Using a separatory funnel and washing 3 times with $100 \mathrm{ml}$ MilliQ water, the precursor is obtained. Heptane is removed by systematically lowering the pressure in a rotary evaporator until a dark brown viscous liquid is obtained.

The second step is the decomposition of the precursor. A solution is prepared of the iron-oleate precursor $(5.40 \mathrm{~g}), 5.681 \mathrm{ml}$ oleic acid (VWR) and $18 \mathrm{ml}$ 1-octadecene (90\%, Sigma Aldrich). Iron oxide nanoparticles are created by rapidly heating the solution, at a rate of $18.33^{\circ} \mathrm{C}$ per minute, to $320^{\circ} \mathrm{C}$ and refluxing the solution for 2.5 hours.

Afterwards the solution is cooled and washed three times with $200 \mathrm{~mL}$ ethanol. The IONP solution can be magnetically separated from the ethanol. Finally, the nanoparticles were dispersed in heptane $(99 \%$, Fisher Scientific) to obtain a concentration of $100 \mathrm{mg} / \mathrm{mL}$. The solution can be stored at room temperature. However, it was found that the stability of the colloidal solution can be drastically improved by sealing the containers with Parafilm and storing them in the refrigerator.

\subsubsection{Amine functionalization of IONPs}

\subsubsection{Functionalization with BOC-protected amines}


The amine functionalization of the IONPs is done in two steps. First a trimethoxysiloxane is synthesized using thiol-ene click chemistry and afterwards the IONPs are functionalized using this molecule.

For the first reaction we have followed a synthetic route described by Tucker-Schwartz et al[23]. A schematic view of the reaction is shown in figure 2. First $314,4 \mathrm{mg}$ of $\mathrm{N}$ BOC-Allylamine (98\%, Acros Organics), $0.372 \mathrm{~mL}$ of (3mercaptopropyl)trimethoxysilane (95\%, Sigma Aldrich) and $51.2 \mathrm{mg}$ DMPAP (99\%, Sigma Aldrich) were added to $2 \mathrm{~mL}$ of chloroform (99.8\%, Acros Organics) in a glass vial. This solution is allowed to react while stirring in the UV-reactor equipped with three LEDs (365 nm, output power $200 \mathrm{~mA}$ ) for 45 minutes.
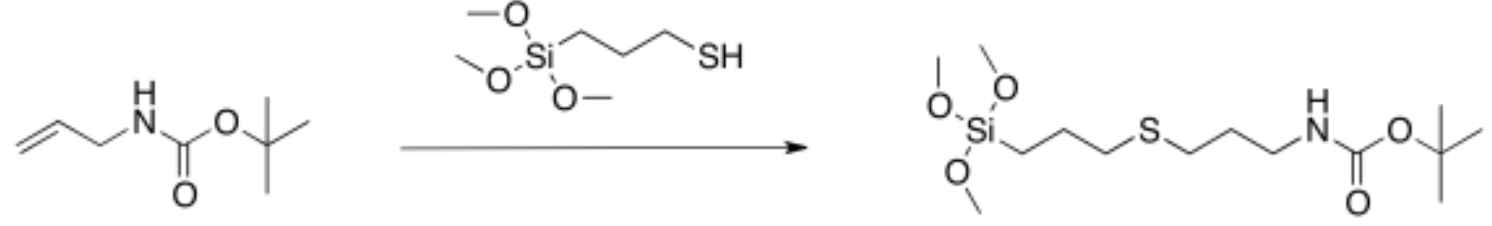

Figure 1: Thiol-ene click chemistry reaction to form the siloxane-protected amine ligand

The second reaction is the functionalization of the IONPs with the silane synthesized with thiol-ene click chemistry, shown schematically in figure 2 . The followed synthetic route is from Bloemen et al. First a solution of $50 \mathrm{~mL}$ toluene (>99.5\%, VWR), $2.5 \mathrm{~mL}$ triethlyamine (99\%, Acros Organics), $2.0 \mathrm{~mL}$ of the IONPs dissolved in heptane (100 $\mathrm{mg} / \mathrm{mL}$ ), $0.05 \mathrm{~mL}$ MilliQ water and $1 \mathrm{~mL}$ of the silane solution was prepared. This solution is then placed in an ultrasonic bath at a temperature of $40{ }^{\circ} \mathrm{C}$ for 5 hours.

Afterwards $50 \mathrm{~mL}$ heptane ( $99 \%$, Fisher Scientific) is added to the obtained solution, which is then placed on a magnet array to isolate the nanoparticles. The nanoparticles are washed with acetone, which is repeated three times. The obtained solid is then dissolved in chloroform to obtain a concentration of $10 \mathrm{mg} / \mathrm{ml}$.

\subsubsection{Deprotection of BOC-protected amines}

To increase the shelf-life of the functionalized nanoparticles, a protective tertbutyloxycarbonyl(BOC)-group is attached to the amine. For subsequent steps in the $\mathrm{Fe}_{3} \mathrm{O}_{4} @ A u$ core-shell nanoparticle synthesis, it is necessary to remove this protective group.

This deprotection is done by adding $20 \mathrm{ml}$ of the previously synthesized BOC-protected amine functionalized IONPs in chloroform $(10 \mathrm{mg} / \mathrm{ml})$ to a solution of $30 \mathrm{ml}$ dichloromethane (analytical reagent grade, Fisher Scientific) and $50 \mathrm{ml}$ trifluoroacetic acid (>99.9\%, Roth) in an Erlenmeyer flask. Then this flask is subsequently sealed with a glass cap and a layer of Parafilm. It is then placed on a shaker at moderate speed at room temperature to react overnight. Afterwards, the flask is placed on a magnet array for magnetic separation. The remaining nanoparticles are washed three times with acetone. Finally, the amine functionalized nanoparticles can be re-dissolved in $10 \mathrm{ml}$ MilliQ water.

\subsection{3 $\mathrm{Fe}_{3} \mathrm{O}_{4} @ A u$ core-shell nanoparticles}

\subsubsection{Direct reduction of gold in ultrasonic bath}

The synthesis is inspired by a method that was used to deposit silver shells onto gold nanoparticles[24]. $1 \mathrm{ml} 37 \%$ formaldehyde solution in $\mathrm{H}_{2} \mathrm{O}$ is added to $20 \mathrm{ml}$ of milli-Q 
water in a closed small glass vial. This vial is left to age for 30 minutes at room temperature. After aging, it is added to $10 \mathrm{ml}$ of amine-functionalized iron oxide nanoparticles solution in a $250 \mathrm{ml}$ glass Erlenmeyer flask. $2,5 \mathrm{ml}$ of a $1 \mathrm{M}$ ascorbic acid solution is also added. This mixture is shaken briefly and left to incubate for 5 minutes. After the incubation, $1-7 \mathrm{ml}$ of a $5 \mathrm{mM} \mathrm{HAuCl}_{4}$ solution is added in its entirety. The solution obtains a dark brown color. The glass flask is subsequently placed in an ultrasonic bath for 2 hours. As the reaction progresses, the color can be observed to change from dark brown to a very dark green and eventually a deep blue when the reaction is close to completion. To remove any remaining reagents and impurities, as well as non-magnetic nanoparticles, the glass flask is placed on a neodymium magnet array to attract the magnetic nanoparticles. After several hours, depending on the thickness of the gold shell, the nanoparticles will aggregate on the bottom and the solvent can be removed. The dry nanoparticles can then be re-dissolved in milli- $Q$ water or 1,4-dioxane. Additional practical information can be found in supporting material S3.

Yields between 4 and $10 \mathrm{mg}$ can be achieved, depending on the amount of gold salt added. Typically, virtually all the gold chloride added is reduced to gold(0) and after magnetic separation, a clear solution containing no non-magnetic gold nanoparticles is obtained. As an example, when $5 \mathrm{ml} 5 \mathrm{mM} \mathrm{HAuCl}_{4}$ was added to $2 \mathrm{mg}$ deprotected amine functionalized iron oxide nanoparticles, a yield of $6.7 \mathrm{mg}$ of core-shell nanoparticles was obtained. The theoretical ideal yield is $6.92 \mathrm{mg}$, resulting in $97 \%$ efficiency and a very small amount of product lost.

\subsubsection{Functionalization of $\mathrm{Fe}_{3} \mathrm{O}_{4} @ A$ Au core-shell nanoparticles with thiolated PEG}

Functionalizing the $\mathrm{Fe}_{3} \mathrm{O}_{4} @ \mathrm{Au}$ core-shell nanoparticles with thiolated PEG will increase the solubility in water, as well as potentially lowering the nanotoxicity. A solution of poly(ethylene glycol) methyl ether thiol (Mn 800 , Sigma Aldrich) of $1 \mathrm{mg} / \mathrm{ml}$ in MilliQ water is prepared. A mass ratio of PEG-thiol to core-shell nanoparticles of 2 is required to ensure an excess of PEG-thiol. In a typical synthesis, $2 \mathrm{ml}$ of $1 \mathrm{mg} / \mathrm{ml}$ PEG-thiol solution is added to $1 \mathrm{ml}$ of $1 \mathrm{mg} / \mathrm{ml} \mathrm{Fe}_{3} \mathrm{O}_{4} @ A u$ core-shell nanoparticles in water. This mixture is placed on a vortex shaker to shake vigorously for 5 minutes. Afterwards, it is placed in an ultrasonic bath for 1 hour. The solution is then placed on a magnet array for up to 2 days for magnetic separation. The nanoparticles take a significant amount of time to be drawn to the magnet due to the high affinity for water of the PEG-ligands. In case of time restraints, it is possible to add a poor solvent like ethanol to speed up the precipitation process. The structure of the PEG-ligands protects the core-shell nanoparticles from aggregation through steric hindrance.

\subsection{Equipment and characterization}

UV-Visible light spectra were collected using a Perkin-Elmer UV-Vis-NIR spectrophotometer Lambda 900. The ultrasonication bath used in both functionalization and the core-shell synthesis was a Bransonic Model 5510 sonicator with a capacity of $10 \mathrm{~L}$. The UV chamber used in the siloxane functionalization is equipped with 3 LEDs ( $365 \mathrm{~nm}$ ) with an output power of $200 \mathrm{~mW}$. For the TEM images, an $80 \mathrm{kV}$ Zeiss EM-900 electron microscope was used in combination with 300 mesh Formvar coated copper grids from Ted Pella. Statistical distribution data were obtained by ImageJ. Magnetic properties were characterized by a superconducting quantum 
interference device (SQUID) magnetometer (by LOT-Quantum Design MPMS 3, San Diego, CA, USA) in dry state.

\section{Results and discussion}

\subsection{Morphology of Fe304@Au nanoparticles}

Since an excess of formaldehyde and ascorbic acid is used in this synthesis, varying the amount of gold salt added will have a direct effect on the amount of gold deposited on the iron oxide nanoparticles. This allows tunability of the nanoparticle size and was studied by making different batches of $\mathrm{Fe}_{3} \mathrm{O}_{4} @ A u$ nanoparticles with 1, 3, 5 and $7 \mathrm{ml}$ of $5 \mathrm{mM} \mathrm{HAuCl}_{4}$ solution added respectively. The effect on the morphology was studied using TEM, and the change in optical properties by UV-Vis spectrometry.

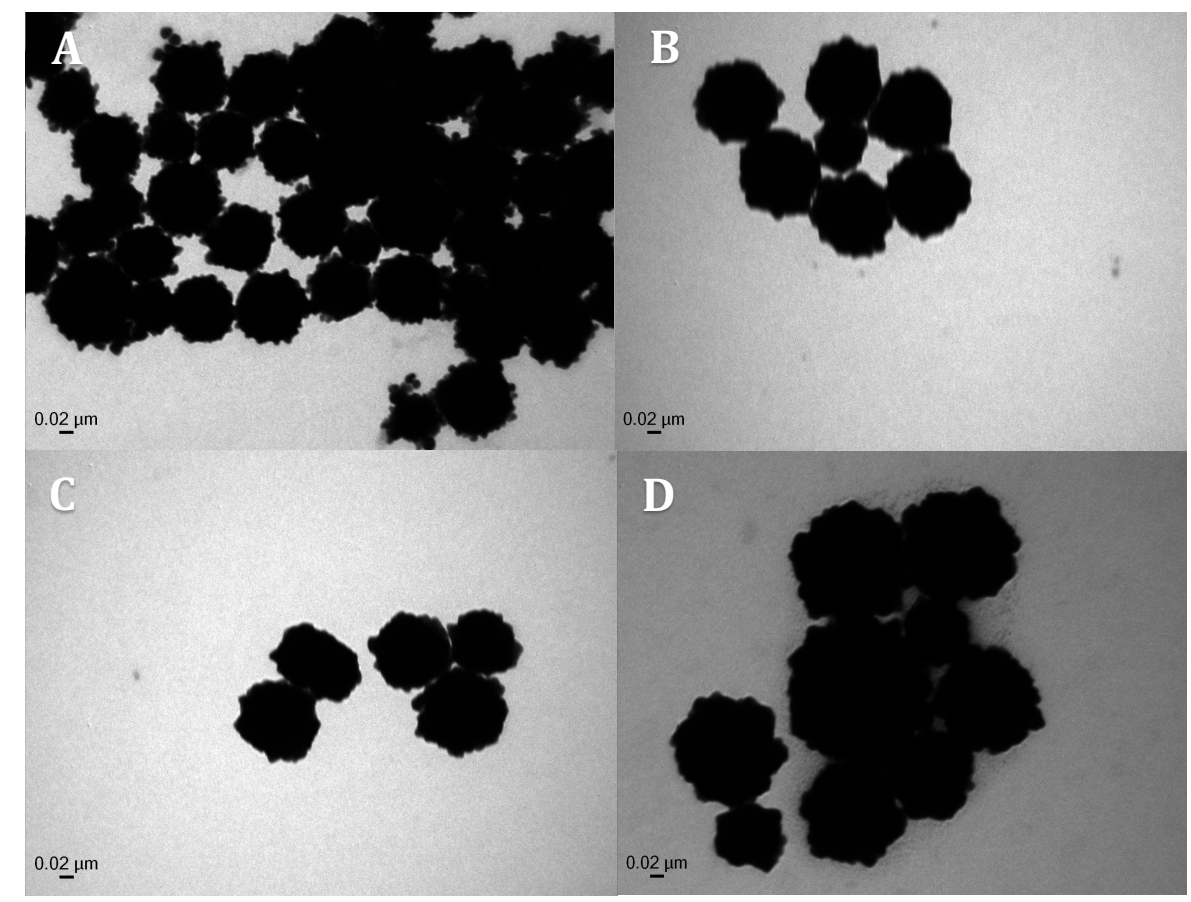

Figure 2: TEM images of core-shell nanoparticles synthesized using a) $1 \mathrm{ml} \mathrm{b)} 3 \mathrm{ml} \mathrm{c)} 5 \mathrm{ml}$ and d) $7 \mathrm{ml} \mathrm{HAuCl}_{4}$

\begin{tabular}{ccc}
\hline $\begin{array}{c}\text { Amount of gold salt solution } \\
\text { added (ml) }\end{array}$ & $\begin{array}{c}\text { Average } \\
\text { size (nm) }\end{array}$ & $\begin{array}{c}\text { Average shell } \\
\text { thickness (nm) }\end{array}$ \\
\hline $\mathbf{1 ~} \mathbf{~} \mathbf{l}$ & $80 \pm 6$ & $30 \pm 3$ \\
\hline $\mathbf{3} \mathbf{~ m l}$ & $97 \pm 10$ & $39 \pm 5$ \\
\hline $\mathbf{5} \mathbf{~ m l}$ & $100 \pm 4$ & $40 \pm 2$ \\
\hline $\mathbf{7} \mathbf{~ m l}$ & $160 \pm 20$ & $70 \pm 10$ \\
\hline
\end{tabular}

Table 1: Statistical analysis of core-shell diameters and shell thickness as a function of the amount of gold salt added

Figure 3 shows TEM images of $\mathrm{Fe}_{3} \mathrm{O}_{4} @ A u$ core-shell nanoparticles that have been synthesized using different amounts of $5 \mathrm{mM} \mathrm{HAuCl}_{4}$ gold salt solution. The images correspond to a) $1 \mathrm{ml} \mathrm{b)} 3 \mathrm{ml} \mathrm{c)} 5 \mathrm{ml}$ and d) $7 \mathrm{ml}$ of gold salt solution added. The mean diameters of the nanoparticles are $80,97,100$ and $160 \mathrm{~nm}$ respectively. Since the 
average iron oxide core diameter is known, the average shell thickness can be calculated. This data is shown in table 1 . It is clear the size of the core-shell nanoparticles can be tuned by means of varying the amount of gold salt added. The apparent roughness of samples a) and b) may indicate incomplete coverage of the gold shell. The roughness in these samples might also be an indication of in-situ generation of gold seeds, rather than the direct deposition of a smooth gold shell. This also implies the gold is not deposited epitaxially. Further evidence of the in-situ generation of gold seeds and subsequent growth is shown in supporting information S6. Though there is a large relative difference in electron density between iron oxide and gold, it is not possible to distinguish an almost-closed from a completely closed gold shell, simply due to the sheer electron density of gold. This renders the core-shells completely black in TEM-images.

\subsection{Optical properties of $\mathrm{Fe}_{3} \mathrm{O}_{4} @ \mathrm{Au}$ nanoparticles}

To study the plasmon resonance of the synthesized core-shell nanoparticles, UV-Vis spectra were recorded. In figure 4, UV-Vis spectra are shown for an array of core-shell nanoparticles.

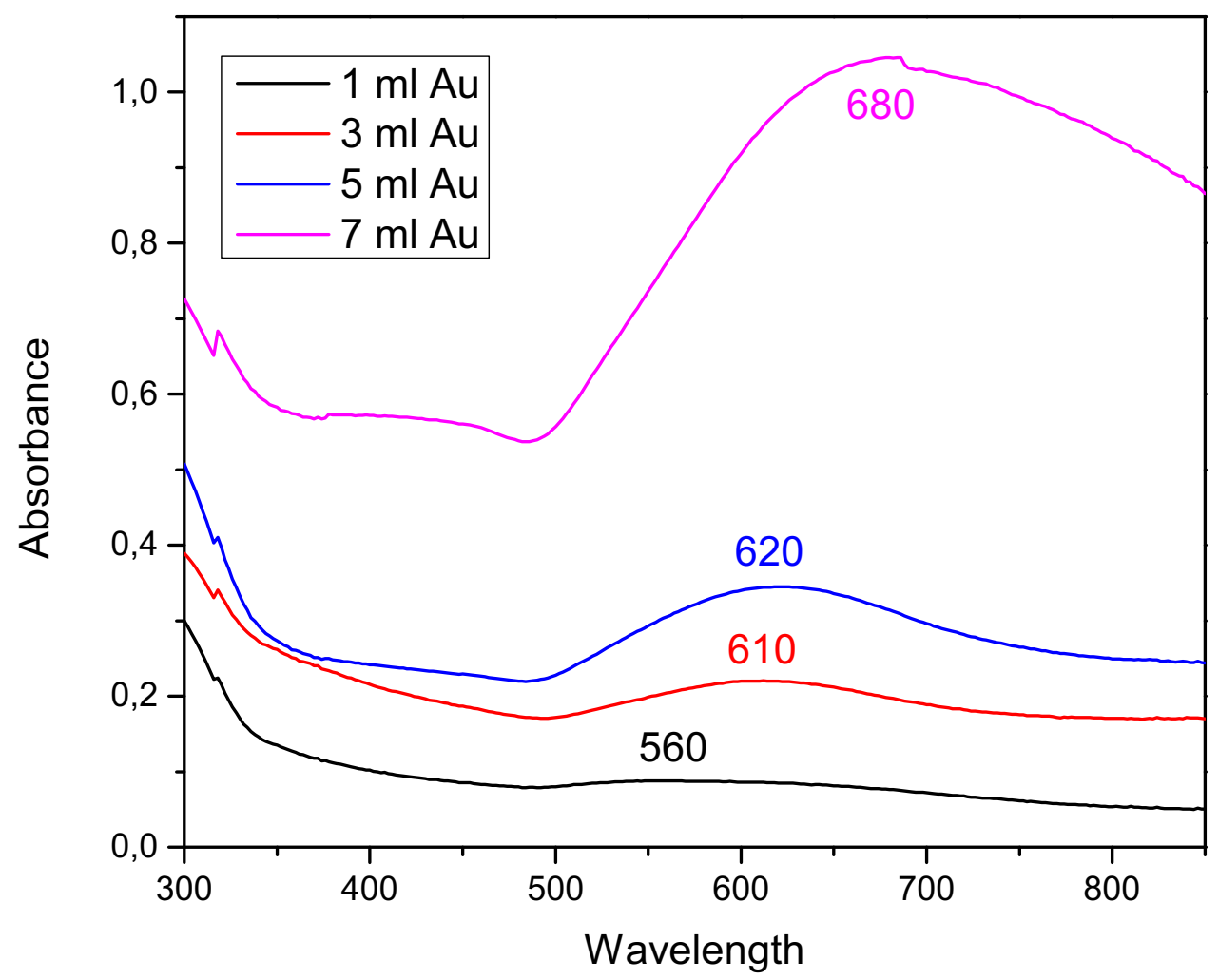

Figure 3: UV-Vis spectrum of core-shells synthesized using (black) $1 \mathrm{ml}$ (red) $3 \mathrm{ml}$ (blue) $5 \mathrm{ml}$ and (fuchsia) $7 \mathrm{ml}$ of $\mathrm{HAuCl}_{4}$

The frequency of plasmonic resonance and therefore the wavelength of the plasmon peak is dependent on the thickness of the plasmonic shell. The plasmon peak can be 
tailored by the amount of gold that is used in the synthesis. For core-shell structures with a thin plasmonic shell, meaning thinner than $15 \mathrm{~nm}$, an increase in the gold shell thickness should be accompanied by a blueshift of the plasmon peak[2]. However, larger core-shell structures will show a redshift with increasing shell thickness due to electric retardation effects[25,26]. The electromagnetic field cannot penetrate beyond a certain depth inside the metal, the so-called skin depth, which is around $15 \mathrm{~nm}$ in the vis-NIR. As a result, the plasmon resonance of the core-shell nanoparticles behaves more like a large pure gold nanoparticle. A size increase will therefore accompany a redshift of the extinction maximum, as observed for gold nanoparticles[6,27,28].

This effect can be observed as the plasmon peak shifts from 560 to $680 \mathrm{~nm}$ with increasing amounts of gold used in the synthesis. For producing stable core-shells, 5 $\mathrm{ml}$ was found to be the ideal amount of gold salt added. Lower amounts may lead to incomplete coverage of the iron oxide, which, since it is functionalized with amines, will have a strong affinity for the gold shells of other particles. This causes crosslinking and results in coagulation. Conversely, adding more than $5 \mathrm{ml}$ of gold salt leads to the formation of very large core-shell structures. The sheer weight of these core-shells causes the effect of gravity to overcome the stabilization of possible ligands, causing the core-shells to precipitate to the bottom of the solution over the course of a few hours. They can however still be redispersed by ultrasonication.

\subsection{Functionalization of $\mathrm{Fe}_{3} \mathrm{O}_{4} @ A u$ core-shell nanoparticles with thiolated PEG}

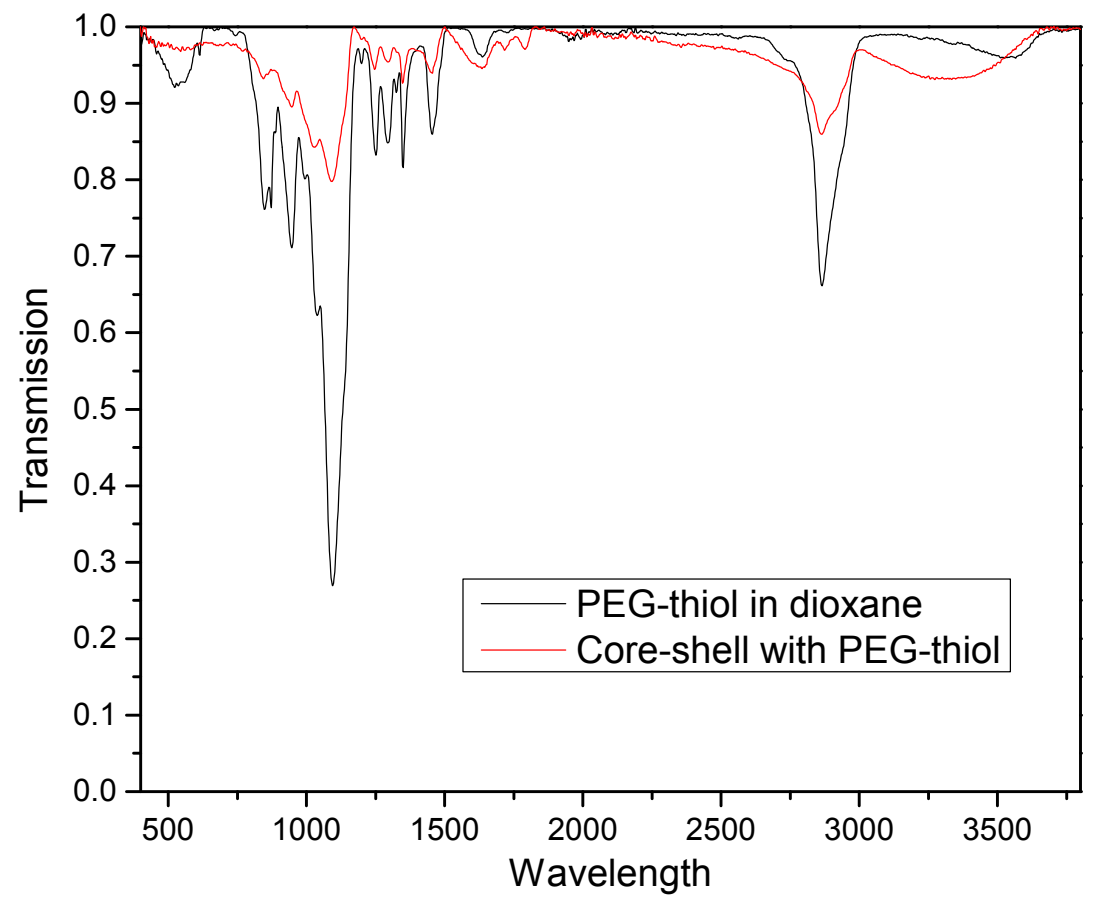

Figure 4: FT-IR spectrum in dioxane of (black) PEG-thiol and (red) $\mathrm{Fe}_{3} \mathrm{O}_{4} @ A$ Au core-shell nanoparticles functionalized with PEG-thiol

$\mathrm{Fe}_{3} \mathrm{O}_{4} @$ Au core-shell nanoparticles were functionalized with a thiolated PEG. Whether the PEG-thiol functionalization was successful can be determined by FT-IR. This is shown in Figure 5, where the black line corresponds to a concentrated solution of PEGthiol in 1,4 dioxane. The red line corresponds to $\mathrm{Fe}_{3} \mathrm{O}_{4} @ \mathrm{Au}$ core-shell nanoparticles 
functionalized with PEG-thiol in 1,4 dioxane. The $\mathrm{C}-\mathrm{H}$ stretch of the PEG-thiol is strongly present in both samples at $2860 \mathrm{~cm}^{-1}$. The same is true for the $\mathrm{O}-\mathrm{H}$ and $\mathrm{C}-\mathrm{O}-$ $\mathrm{H}$ stretch at $1094 \mathrm{~cm}^{-1}$. Other less prominent peaks of PEG-thiol also seem to be present in the sample of functionalized core-shells. The spectrum of the functionalized core-shells shows no prominent peaks at 580-600 and 470 to $480 \mathrm{~cm}^{-1}$, which would indicate the presence of iron oxide. The absence of these peaks is additional confirmation that the gold-shell is closed and no iron oxide is exposed. It can be concluded that the PEG-thiol functionalization has been successful and closed gold shells have been synthesized.

\subsection{Magnetic properties of synthesized nanoparticles and core-shells}

The magnetic moment of iron oxide nanoparticles as synthesized is shown in supporting material S4.

The iron oxide nanoparticles coated with oleic acid, as synthesized, reach a saturation magnetization of $55 \mathrm{emu} / \mathrm{g}$. This is comparable to the $67.4 \mathrm{emu} / \mathrm{g}$ that was measured by Chen for iron oxide nanoparticles $20.4 \mathrm{~nm}$ in size[20]. This, coupled with the lack of remnant magnetization, is another indication of a successful synthesis of superparamagnetic iron oxide nanoparticles.

To further determine the existence of a magnetic core and to study the influence of increasing gold shell size on the magnetic behavior, SQUID measurements were taken of the same array of samples as shown earlier in the TEM images. These are shown in figure 6.

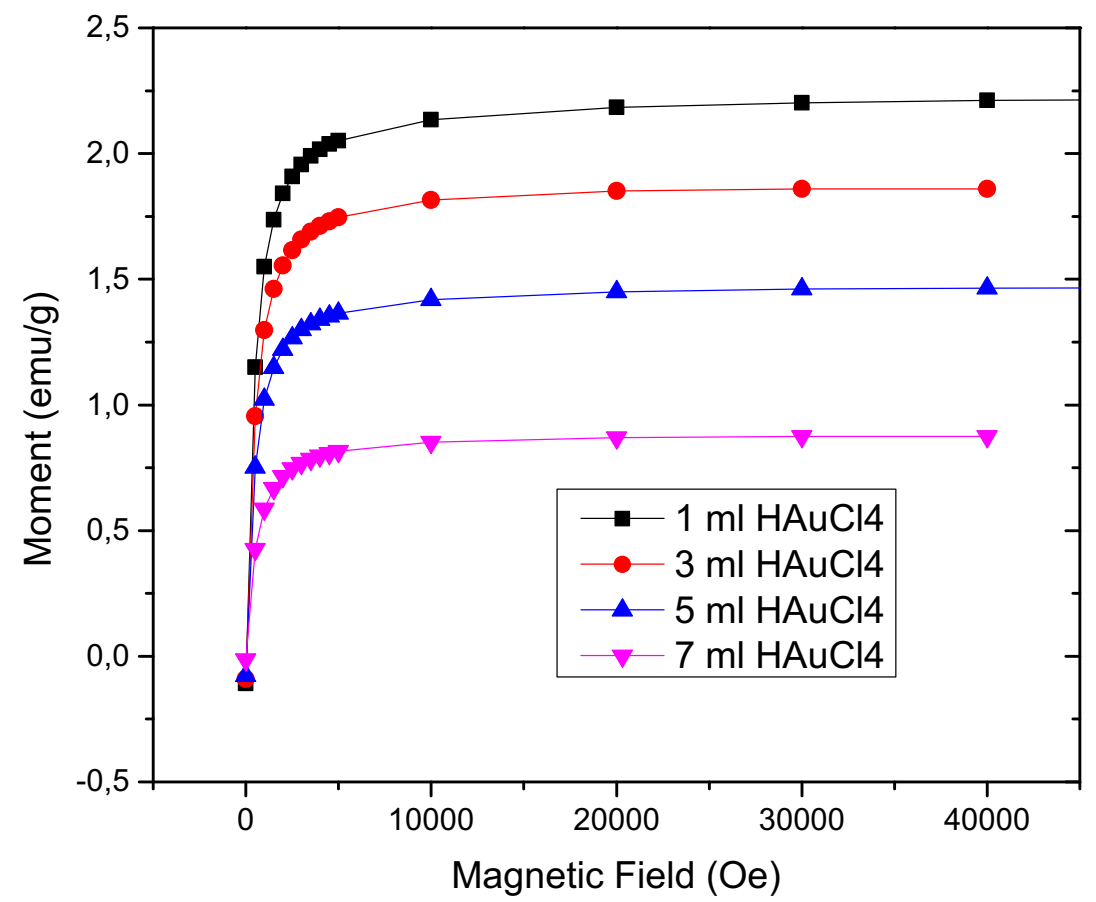

Figure 6: SQUID magnetism measurement of $\mathrm{Fe}_{3} \mathrm{O}_{4} @ A$ core-shell nanoparticles synthesized using (black) $1 \mathrm{ml}$ (red) $3 \mathrm{ml}$ (blue) $5 \mathrm{ml}$ and (fuchsia) $7 \mathrm{ml} \mathrm{HAuCl}_{4}$ 
Relative to the IONPs as synthesized saturation magnetization of $55 \mathrm{emu} / \mathrm{g}$, a decrease to $2.25 \mathrm{emu} / \mathrm{g}$ is noted for the thinnest of gold shells synthesized. Also, there is a clear downward trend in saturation magnetization as the thickness of the gold shell increases. This is as expected, since there are 2 acting effects; first the increased thickness of the gold shell, which is diamagnetic, will increase the magnetic shielding the superparamagnetic core experiences from the diamagnetic gold. A second effect is that the ratio of the mass of gold to iron oxide increases, which will logically decrease saturation magnetization per gram.

To demonstrate the magnetic behavior in practice, a sample of core-shells dissolved in water were placed next to a neodymium magnet. This is shown in supporting material S5. It is shown that even when dispersed in a good solvent, the $\mathrm{Fe}_{3} \mathrm{O}_{4} @ \mathrm{Au}$ core-shell nanoparticles can still be extracted magnetically, which makes them very practical to use in a multitude of applications and experimental steps.

\subsection{Approximation of the number of magnetic cores per core-shell nanoparticle}

By comparing the expected magnetic force to the measured magnetic force the $\mathrm{Fe}_{3} \mathrm{O}_{4} @ \mathrm{Au}$ core-shell nanoparticles exhibit, an estimate can be made for the number of magnetic cores per core-shell nanoparticle. For the thinnest gold shell deposited, the saturation magnetization has decreased by a factor of 6.66 relative to prior to the gold shell deposition. If the nanoparticle is considered to have a single magnetic core of $20 \mathrm{~nm}$, the weight of the $30 \mathrm{~nm}$ thick gold shell is already approximately 200 times that of the magnetic core. By weight alone, the saturation magnetization of a single magnetic core nanoparticle should therefore be considerably lower, $0.08 \mathrm{emu} / \mathrm{g}$ in this case compared to the measured value of $2.25 \mathrm{emu} / \mathrm{g}$. This indicates the presence of multiple magnetic cores per core-shell nanoparticle.

By comparing the magnetic force and the sizes of the core-shell nanoparticles, an average amount of cores per core-shell nanoparticle can be calculated. Since the radius of the core-shell nanoparticle and the magnetic core is known, a number of cores can be found where the fraction of gold to magnetite equals the decrease in magnetic force. The weight of gold that is removed when additional magnetic cores are added is accounted for. For the samples made using 1, 3, 5 and $7 \mathrm{ml}$ of $\mathrm{HAuCl}_{4}$ this yields an average of $8,13,11$ and 18 cores per nanoparticle, respectively. $A$ general trend towards a higher number of magnetic cores with increasing gold content can be observed. Higher amounts of gold deposited seems to increase the chances of clustering of magnetic cores.

These numbers should be considered broad approximations since at the moment it is very difficult to look inside a core-shell nanoparticle coated with a relatively thick layer of gold to get an actual reading of the number of magnetic cores. Also, since this calculation looks at relative weight fractions only, the additional effect of magnetic shielding by diamagnetic materials has not been taken into account.

Supporting information S7 shows a TEM-EDX analysis of a single core-shell nanoparticle which shows that Fe-atoms are present inside the Au-shell, however, the resolution does not allow for distinguishing singular magnetic cores as the Fe appears to be relatively evenly distributed across the nanostructure. This is an additional confirmation of the presence of magnetic material inside the gold shell.

\section{Conclusion}


Iron oxide-gold core-shell nanoparticles were successfully synthesized using a novel method. The gold shell was formed by reduction of gold salt in an ultrasonic bath. Optical characteristics were studied using UV-Vis spectroscopy. The morphology was examined using TEM. The presence of the magnetic core was determined by SQUIDmagnetometry. Further evidence that the gold shell is completely closed is provided by FT-IR. The presence of iron inside the gold shell is confirmed by TEM-EDX. The thickness of the gold shell could be tuned by controlling the amount of gold salt added. With increased gold salt amounts, the shell thickness increased, as well as the estimated number of magnetic cores per core-shell nanoparticle. To emphasize the value for biomedical applications and the ease of functionalization, the functionalization of the core-shells with PEG-thiol was successfully executed and confirmed.

\section{Acknowledgements}

We acknowledge financial support from the Agency for Innovation and Technology in Flanders (IWT). We also acknowledge financial support from the Special Research Fund (BOF) of the Flemish community as a C3 project. We would also like to thank the Hercules Foundation for the funding of the SQUID magnetometer.

\section{Conflict of interest}

The authors declare no conflict of interest.

\section{References}

[1] M. Bloemen, W. Brullot, T.T. Luong, N. Geukens, A. Gils, T. Verbiest, Improved functionalization of oleic acid-coated iron oxide nanoparticles for biomedical applications., J. Nanopart. Res. 14 (2012) 1100. doi:10.1007/s11051-0121100-5.

[2] W. Brullot, V.K. Valev, T. Verbiest, Magnetic-plasmonic nanoparticles for the life sciences: calculated optical properties of hybrid structures, Nanomedicine Nanotechnology, Biol. Med. 8 (2012) 559-568. doi:10.1016/j.nano.2011.09.004.

[3] R. Qiao, C. Yang, M. Gao, Superparamagnetic iron oxide nanoparticles: from preparations to in vivo MRI applications, J. Mater. Chem. 19 (2009) 62746293. doi:10.1039/b902394a.

[4] I.-I.S. Lim, P.N. Njoki, H.-Y. Park, X. Wang, L. Wang, D. Mott, C.-J. Zhong, Gold and magnetic oxide/gold core/shell nanoparticles as bio-functional nanoprobes., Nanotechnology. 19 (2008) 305102. doi:10.1088/09574484/19/30/305102.

[5] X. Huang, M. a. El-Sayed, Gold nanoparticles: Optical properties and implementations in cancer diagnosis and photothermal therapy, J. Adv. Res. 1 (2010) 13-28. doi:10.1016/j.jare.2010.02.002.

[6] J. Kimling, M. Maier, B. Okenve, V. Kotaidis, H. Ballot, A. Plech, Turkevich Method for Gold Nanoparticle Synthesis Revisited, (2006) 15700-15707.

[7] J. Zhou, J. Ralston, R. Sedev, D.A. Beattie, Functionalized gold nanoparticles: Synthesis, structure and colloid stability, J. Colloid Interface Sci. 331 (2009) 251-262. doi:10.1016/j.jcis.2008.12.002. 
[8] A.M. Alkilany, C.J. Murphy, Toxicity and cellular uptake of gold nanoparticles: what we have learned so far?, (2010) 2313-2333. doi:10.1007/s11051-0109911-8.

[9] G. Von Maltzahn, J.H. Park, A. Agrawal, N.K. Bandaru, S.K. Das, M.J. Sailor, S.N. Bhatia, Computationally guided photothermal tumor therapy using longcirculating gold nanorod antennas, Cancer Res. 69 (2009) 3892-3900. doi:10.1158/0008-5472.CAN-08-4242.

[10] F. Pene, E. Courtine, A. Cariou, J.-P. Mira, Toward theragnostics, Crit. Care Med. 37 (2009) S50-S58. doi:10.1097/CCM.0b013e3181921349.

[11] P. Gangopadhyay, S. Gallet, E. Franz, a. Persoons, T. Verbiest, Novel superparamagnetic Core(Shell) nanoparticles for magnetic targeted drug delivery and hyperthermia treatment, IEEE Trans. Magn. 41 (2005) 41944196. doi:10.1109/TMAG.2005.854805.

[12] B.K. Sodipo, A.A. Aziz, M. Mustapa, Facile synthesis and characteristics of gold coated superparamagnetic iron oxide nanoparticles via sonication, 8 (2015) 1-6.

[13] X. Ji, R. Shao, A.M. Elliott, R. Jason Stafford, E. Esparza-Coss, J. a. Bankson, G. Liang, Z.P. Luo, K. Park, J.T. Markert, C. Li, Bifunctional Gold Nanoshells with a Superparamagnetic Iron Oxide-Silica Core Suitable for Both MR Imaging and Photothermal Therapy, J. Phys. Chem. C. 111 (2007) 6245-6251. doi:10.1021/jp0702245.

[14] I.Y. Goon, L.M.H. Lai, M. Lim, P. Munroe, J.J. Gooding, R. Amal, Fabrication and Dispersion of Gold-Shell-Protected Magnetite Nanoparticles : Systematic Control Using Polyethyleneimine, 9 (2009) 673-681.

[15] Z. Ban, Y. a. Barnakov, F. Li, V.O. Golub, C.J. O'Connor, The synthesis of core-shell iron@gold nanoparticles and their characterization, J. Mater. Chem. 15 (2005) 4660. doi:10.1039/b504304b.

[16] J.E. Araújo, C. Lodeiro, J.L. Capelo, B. Rodríguez-González, A.A. dos Santos, H.M. Santos, J. Fernández-Lodeiro, Novel nanocomposites based on a strawberry-like gold- coated magnetite (Fe@Au) for protein separation in multiple myeloma serum samples, Nano Res. 8 (2015) 1189-1198. doi:10.1007/s12274-014-0599-4.

[17] Q. Zhang, J. Ge, J. Goebl, Y. Hu, Y. Sun, Y. Yin, Tailored synthesis of superparamagnetic gold nanoshells with tunable optical properties, Adv. Mater. 22 (2010) 1905-1909. doi:10.1002/adma.200903748.

[18] L. Wang, J. Luo, Q. Fan, Monodispersed core-shell Fe3O4@ Au nanoparticles, J. .... (2005) 21593-21601.

[19] I.-C. Chiang, D.-H. Chen, Structural characterization and self-assembly into superlattices of iron oxide-gold core-shell nanoparticles synthesized via a hightemperature organometallic route., Nanotechnology. 20 (2009) 015602 1-7. doi:10.1088/0957-4484/20/1/015602.

[20] C.J. Chen, H.Y. Lai, C.C. Lin, J.S. Wang, R.K. Chiang, Preparation of monodisperse iron oxide nanoparticles via the synthesis and decomposition of iron fatty acid complexes, Nanoscale Res. Lett. 4 (2009) 1343-1350. doi:10.1007/s11671-009-9403-x.

[21] C.W.V. Gmbh, C. Kgaa, M. Bloemen, B. Sutens, W. Brullot, A. Gils, N. Geukens, Two-Step Directional Surface Modification of Iron Oxide Nanoparticles with Protected Siloxanes, (2015).

[22] J. Park, K. An, Y. Hwang, J.-G. Park, H.-J. Noh, J.-Y. Kim, J.-H. Park, N.-M. Hwang, T. Hyeon, Ultra-large-scale syntheses of monodisperse nanocrystals., 
Nat. Mater. 3 (2004) 891-5. doi:10.1038/nmat1251.

[23] A.K. Tucker-Schwartz, R.A. Farrell, R.L. Garrell, Thiol - Ene click reaction as a general route to functional trialkoxysilanes for surface coating applications, J. Am. Chem. Soc. 133 (2011) 11026-11029. doi:10.1021/ja202292q.

[24] C.S. Nanoparticles, C.B. Norris, P.R. Joseph, M.R. Mackiewicz, S.M. Reed, Minimizing Formaldehyde Use in the Synthesis of Gold - Silver, (2010) 3637 3645. doi:10.1021/cm9035693.

[25] E. Ringe, M.R. Langille, K. Sohn, J. Zhang, J. Huang, C.A. Mirkin, R.P. Van Duyne, L.D. Marks, Plasmon length: A universal parameter to describe size effects in gold nanoparticles, J. Phys. Chem. Lett. 3 (2012) 1479-1483. doi:10.1021/jz300426p.

[26] V. Myroshnychenko, J. Rodríguez-Fernández, I. Pastoriza-Santos, A.M. Funston, C. Novo, P. Mulvaney, L.M. Liz-Marzán, F.J. García De Abajo, Modelling the optical response of gold nanoparticles, Chem. Soc. Rev. 37 (2008) 1792-1805. doi:10.1039/b711486a.

[27] M. Wuithschick, A. Birnbaum, S. Witte, M. Sztucki, U. Vainio, N. Pinna, K. Rademann, F. Emmerling, R. Kraehnert, J. Polte, Turkevich in New Robes: Key Questions Answered for the Most Common Gold Nanoparticle Synthesis, ACS Nano. 9 (2015) 7052-7071. doi:10.1021/acsnano.5b01579.

[28] P. Dobrowolska, A. Krajewska, M. Gajda-Rączka, B. Bartosewicz, P. Nyga, B. Jankiewicz, Application of Turkevich Method for Gold Nanoparticles Synthesis to Fabrication of SiO2@Au and TiO2@Au Core-Shell Nanostructures, Materials (Basel). 8 (2015) 2849-2862. doi:10.3390/ma8062849.

[29] J. Clinton, L. Liebermann, Dead layers in Ferromagnetic Transition Metals, Phys. Rev. Lett. 25 (1970) 232-235. 